\title{
Enhancing computerized cognitive rehabilitation with 3D solutions
}

\author{
Anna Alloni, Silvia Panzarasa, \\ Silvana Quaglini ${ }^{1}$ \\ Department of Industrial and \\ Information Engineering \\ University of Pavia \\ \{anna.alloni | silvana.quaglini | \\ silvia.panzarasa\}@unipv.it
}

\author{
Chiara Zucchella \\ IRCCS C. Mondino Foundation \\ Pavia, Italy \\ chiara.zucchella@gmail.com
}

\author{
Dani Tost \\ Computer Graphics Division \\ CREB, UPC \\ Barcelona, Spain \\ dani@lsi.upc.edu
}

\begin{abstract}
In recent years, cognitive rehabilitation is shifting from paperbased to computer-based practice.

Computerization entails several advantages, among which the availability of a great amount of stimuli to be used within a wide range of easily customizable exercise types and the possibility to monitor the evolution of a patient's skills through the storage of his performance data into a database. Furthermore, a computerized system can automatically assess the patient and adjust each exercise's difficulty accordingly.

Patients undergoing exercises which fail to entertain them and stimulate their curiosity, are very likely to develop fatigue and boredom; besides, perceiving computerized rehabilitation as stressful and uncomfortable could very likely reduce the subject's compliance. For this reason great efforts are to be made in order to avoid the onset of stress states: this is why we chose to differentiate the exercises as much as possible before testing the tool on volunteers and patients. The results led us to improve the system, focusing mainly on the visual aspect of the exercises and to introduce $3 \mathrm{D}$ technology into our system.
\end{abstract}

\section{Categories and Subject Descriptors}

H.1.2 [Models and Principles]: User/machine systems - human factors, human information processing, software psychology.

\section{General Terms}

Experimentation, Human Factors, Performance, Design.

\section{Keywords}

Computer-aided therapy, Cognitive rehabilitation, 3D graphics, Serious games.

\section{INTRODUCTION}

Cognitive rehabilitation is usually administered in form of exercises, which the patients are expected to execute with pen and paper. In the latest years computer science applications have been developed to support this kind of treatment [Schuhfried 1995; Giunti O.S. 2010; Anastasis 2007; Fernández et al. 2012; Dwolatzky et al. 2004; Kueider et al. 2012; Tornatore et al. 2005]. While these applications are in general well-accepted by young

Permission to make digital or hard copies of all or part of this work for personal or classroom use is granted without fee provided that copies are not made or distributed for profit or commercial advantage and that copies bear this notice and the full citation on the first page. To copy otherwise, to republish, to post on servers or to redistribute to lists, requires prior specific permission and/or a fee. REHAB 2014, May 20-23, Oldenburg, Germany Copyright (C) 2014 ICST 978-1-63190-011-2 DOI 10.4108/icst.pervasivehealth.2014.255358 people or children, that need rehabilitation for example after a trauma or for learning and behavioural problems, the older patients (e.g. post-stroke or with degenerative diseases) may have some problems with them, since third age is a part of the population which normally doesn't show much familiarity with modern technology [Eurostat 2014, Richardson 2002]. Such lack of comfort with PCs and informatics tools often entails negative consequences when it comes to trying to integrate such technology into the therapy routine: feeling this kind of innovation- something new and very likely never seen before, for which they are completely unprepared- "forced" upon them often leads to a decrease in the willingness of the patients to actively participate in the process, to the point of developing some sort of "hostility" towards a tool they are not able to handle with ease.

Beside the matter of old age, also the particular condition of subjects suffering cognitive impairments could make them even more reluctant when confronted with a new type of therapy. All of this could even lead to a decrease of the patient's compliance.

On the other side, the "pen and paper" style in the mid-long term can make the therapy boring for the patients and time-consuming for the psychologists. As a matter of fact, the range of different stimuli (images and texts) available is limited by the (usually small) number of existing variants of the printed exercise sheets, and the therapist must be careful to not repeat the same stimuli within a short time. Moreover, the lack of an automated way of recording the results of the sessions prevents the therapists from monitoring the evolution of the patients' clinical condition with ease.

For this reason, being able to integrate computer science into the rehabilitation path in a way that reduces the potentially negative impact to the patient as much as possible is extremely important. This was the main goal during the development of our system.

\section{METHODS}

The collaboration between the "Mario Stefanelli" Laboratory for Biomedical Informatics of the University of Pavia and the IRCCS Fondazione C. Mondino led to the creation of a software tool for computerized cognitive rehabilitation. The system, named CoRe (from Cognitive Rehabilitation), allows the user (the psychologist) to easily generate a computerized version of exercises aimed at restoring logical and executive brain functions, usually undergone with pen and paper during face to face rehabilitation sessions; besides, since the system keeps track of several performance parameters during the execution of the session, it can provide therapists a great help: by analyzing those parameters, CoRe is able to automatically assess the patient and

\footnotetext{
${ }^{1}$ Author order: Anna Alloni, Dani Tost, Silvia Panzarasa, Chiara Zucchella, Silvana Quaglini.
} 
thus to adjust each exercise's difficulty accordingly. As stated before, patient-tailoring of the exercises plays a vital role in preventing fatigue and boredom, since perceiving the rehabilitation as a stressful situation could very likely reduce the subject's compliance.

\subsection{SYSTEM CHARACTERISTICS}

\section{CoRe features four main components:}

-a Visual Basic interface allowing the user (that is, the therapist who creates the treatment plans for the patients) to set the parameters needed to start an exercise (difficulty level, number and maximum duration of the stimuli to be shown etc.);

-two MS Access databases, used to store the patients' personal information and performance parameters, as well as all the stimuli (texts, sounds and images) needed for the execution of the sessions;

-a software engine, implemented in Visual Basic, able to generate the customized exercises based on both the options set by the therapist and the patient's performance;

-the exercises, which have been created with E-Prime, a commercial tool whose main advantage is the extreme flexibility, that allows a user to easily implement all the types of rehabilitation exercises needed.

The structure of CoRe is shown in Figure 1. Just after setting the parameters, the therapist can start the exercises; the databases are accessed for Create Read Update Delete (CRUD) operations and three main purposes:

-when the database must be updated, and new stimuli inserted;

-when these stimuli need to be extracted in order to generate a new session;

-when the system needs to read/write the patient's performances and personal information.

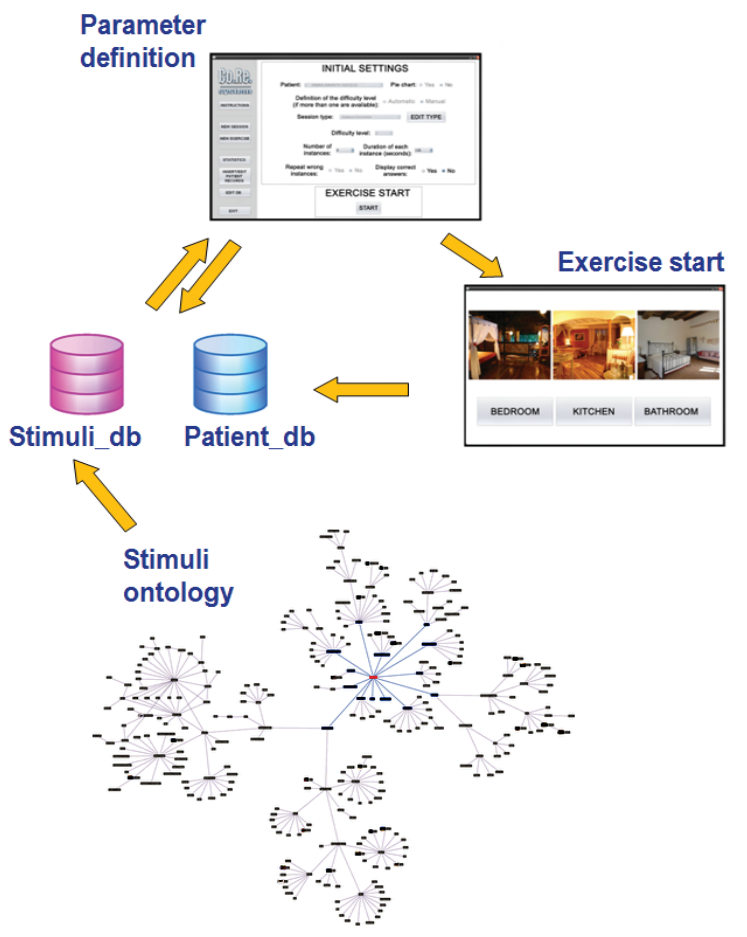

Figure 1. Structure of CoRe.

The exercises featured in the system require the completion of several types of tasks based on the patient correctly recognizing the stimuli proposed. For example, Figure 2 shows an instance of "Pick the element": the patient is shown a matrix of textual stimuli (the numbers visualized in each slot), among which he must choose the one requested by the system (here, the number 0 ).

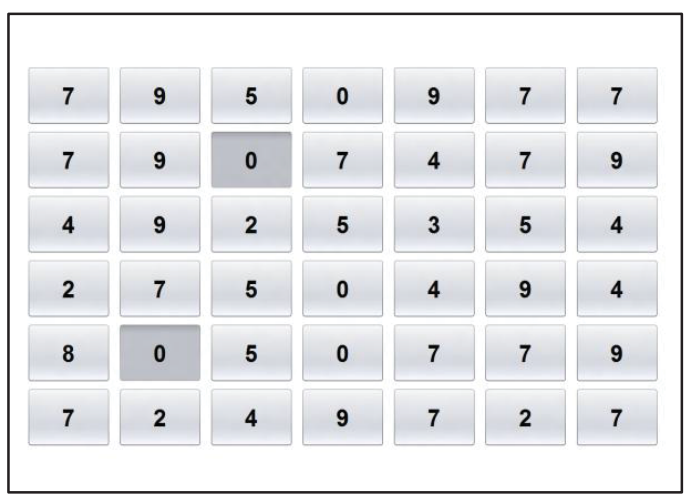

Figure 2. An instance of "Pick the element"

To be able to generate the sessions correctly, the system interacts with a stimuli database containing about 6000 entries- words, images and sounds. This, of course, makes it possible to create exercises dynamically, thus exponentially increasing the number of variants available for execution: it's almost impossible that during an exercise the patient undergoes more instances based on the very same stimuli. This implementation choice- generation of dynamic sessions- represents the first step taken to solve the problem of boredom and prevent non-compliance.

Some tasks also require the user to identify relationships defined between stimuli. This is the case for "Find the intruder", shown in Figure 3: five buttons are shown on screen, each one labeled with a word. Four of them belong to the same category, while one of them does not. The patient's goal is to select the intruder.

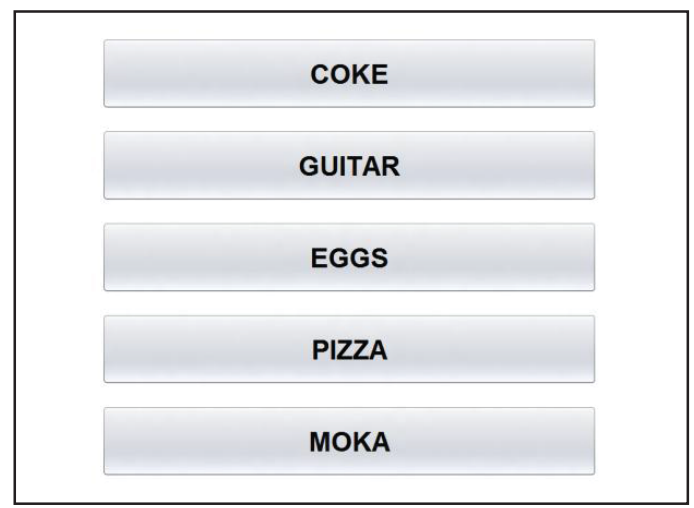

Figure 3. An instance of "Find the intruder"

From a technical point of view, the most effective way of building such system- that is, one able to generate relation-based exercises correctly- is by organizing all the stimuli in an ontology, which describes every element through a set of attributes, and its relations with other entities. For example, the attribute "difficulty level" ("low", "medium", "high") associated to every single concept of the structure and related to the meaning of the concept itself, may be considered initially to retrieve stimuli according to the patient's scholarship (even if exercises will then be adjusted according to the patient's performance). "Is-a" relations among stimuli can instead be used to generate exercises for classification 
tasks. A detailed description of the ontology is beyond the scope of this paper and can be found in [Leonardi et al. 2011].

\section{TEST PHASE AND ISSUES}

The system as described above was first tested by volunteers who underwent simulated rehabilitation sessions in presence of a therapist and then were asked to comment the system: during this phase some issues emerged, that lead us to look for new strategies and solutions to make CoRe as compliant to the patients' needs as possible. Later the system has been tested on 9 Parkinson patients, who were supposed to undergo 12 sessions in a 1-month time range (6 patients performed all the sessions, while 3 were discharged before the end of the trial and dropped out after respectively 9, 10 and 11 sessions). While the general opinion about the system was positive, it was not hard to notice that certain types of tasks were generally preferred to others. In particular, the subjects appeared more entertained and involved when they were asked to solve exercises that featured visual stimuli- for example "Unscramble the images", shown in Figure 4, in which the user has to put a scrambled series of cartoons in the right order to tell a short story.

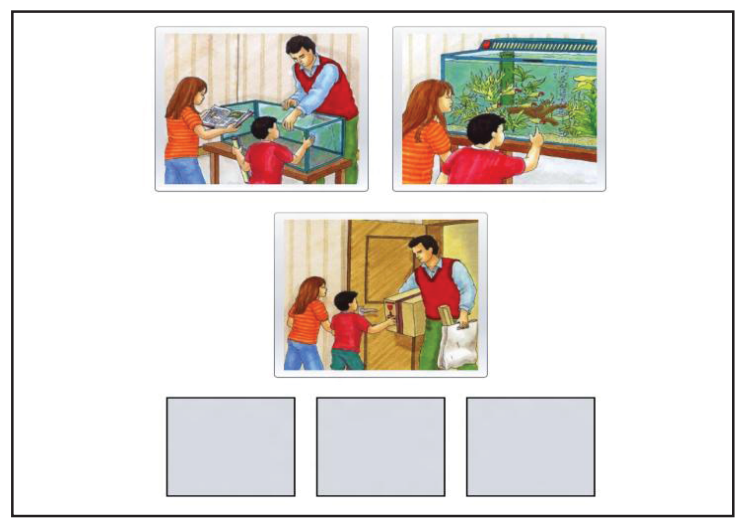

Figure 4. An instance of "Unscramble the images"

This is confirmed by the fact that once the session was over and the volunteers were asked to solve a "text-only" exercise, it was not infrequent to hear them ask "Can't we do some more scene unscrambling instead of this?", even if the new exercise was based on the exact same task, as is the case with "Unscramble the sentence", that requires the user to select words in the right order to form a phrase (Figure 5).

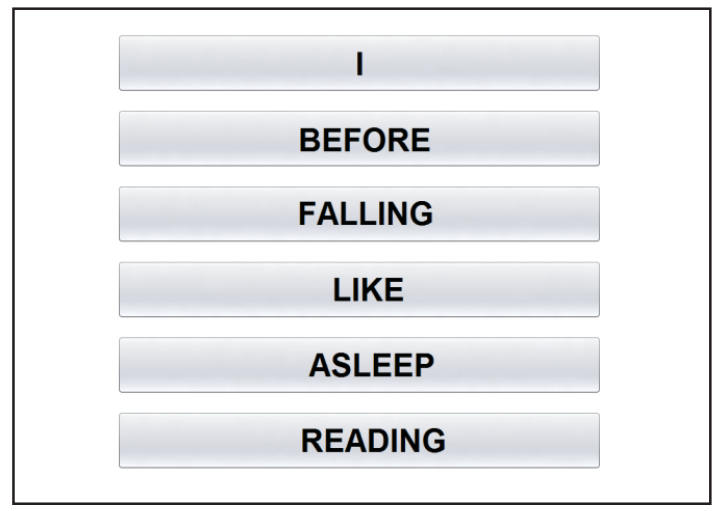

Figure 5. An instance of "Unscramble the sentence"

Among the "text-only" tasks, CoRe features the previously described exercise called "Find the intruder" (Figure 3) that exploits the relations between the concepts represented in the ontology.

Despite being a very simple exercise that requires only one click to be solved, the volunteers didn't seem to enjoy this task very much- for the reason explained above.

The strategy we chose to solve this problem consisted in transforming "Find the intruder" in a visual exercise: contrary to "Unscramble the sentence", in which grammar and syntax play a vital role, this exercise is based on recognition of concepts and thus better suited for reimagining, that can be both $2 \mathrm{D}$ or $3 \mathrm{D}$ based. In the following section we focus on the latter solution.

\section{3D GRAPHICS}

In the latest years, with serious games gaining public attention and becoming an object of interest for researchers, 3D graphics have been introduced in many fields of the medical practice, among which psychological rehabilitation [Abreu et al. 2011; Grau et al. 2010; Rego 2010; Tost et al. 2009; Rizzo et al. 2006].

We chose to develop the visual version of "Find the intruder" as a 3D-based exercise for one main reason: for a human subject a direct interaction with actual, material objects is perceived as more natural than its "abstract" counterpart, that is interacting with bare text (the words that "represent" said objects).

Being the use of (hundreds of) physical items during rehabilitation sessions a clearly non-viable option, the best alternative is setting up an interaction between the user and a visual representation of every object.

This, of course, could be achieved using simple .jpg images easily available online, but the advantages entailed by the use of 3dimensional models are clear: first of all, the use of 3D models allows a more complex interaction, giving users the chance to have a $360^{\circ}$ view of the solids and, in general, to manipulate them at pleasure: secondly, the programmer has complete control over the looks of a model- every single vertex, edge and face of a solid is editable in any moment- something that does not happen with $2 \mathrm{D}$ images.

In the implementation phase, carried out in collaboration with the Computer Graphics Division of the Polytechnical University of Catalonia (UPC), some important matters had to be taken into consideration. First and most important, during the creation of object models a compromise had to be reached between detail and complexity: the more the vertices composing the solid, the bigger the resulting object file and the computational power needed to render the scene. This will be even more important when the time comes to use CoRe and the re-imagined 3D exercises for telerehabilitation sessions: our typical cognitively impaired patients- that is mostly elderly people with very limited familiarity with modern technologies- very likely do not own powerful PCs. Thus it was necessary to keep the system requirements of our software as low as possible. As a thumb rule, we chose to set the maximum number of vertices-per-object to about 10.000 .

Secondly, the 3D version of the exercise features a scenario (a room with 3 bare walls and a floor, with a big table as the only piece of furniture, see Figure 6) that, though very simple and limited in space, the user is allowed to navigate freely, to get as close to the objects as possible.

This, coupled with the possibility to have a $360^{\circ}$ view of the single objects, implied the need of a "complex" control system: it is clear how the simple combination of mouse buttons + keyboard arrows cannot fulfill all the required types of interaction. This is why another compromise is needed, this time between the degrees of freedom allowed to the user and the complexity of the controls. 


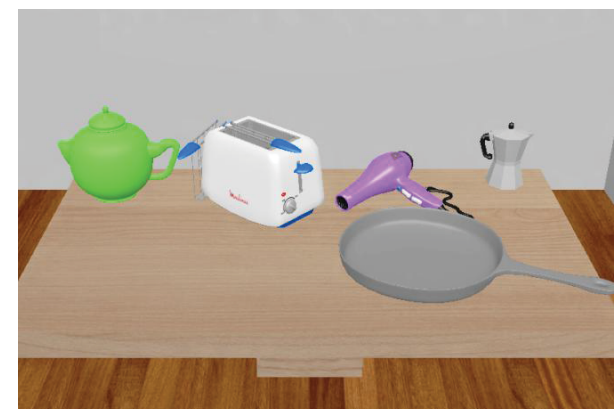

Figure 6. The 3D implementation of "Find the intruder"

\section{DISCUSSION}

Some aspects of our system- especially the ones regarding the new $3 \mathrm{~d}$ features- still need some improvements.

To solve the still open question regarding the creation of a control system simple enough to be handled by cognitively impaired subjects, and at the same time able to provide the navigability and interactivity needed for the execution of the exercise, the introduction of particular hardware solutions must be also taken into consideration: being the manipulation of objects a required feature, the use of a touch screen (maybe even a multi-touch for zooming purposes) could represent an interesting option. Of course, in that case usability tests must be undergone to verify whether this type of control is better accepted than the "classic" (even if slightly more complicated) mouse - keyboard combination.

In case of the default bare scenario not being well accepted by the users, it is also possible to recreate more familiar environments, just by adding textures to the walls and floor of the room. If this feature is accepted, it can also be used to generate different levels of difficulty for the exercise: for example, if the common category is "groceries", the use of a texture representing the aisles of a grocery store might help the patient pinpoint the context and, consequently, identify the intruder faster.

Also, it is not possible to assess which approach (text-only vs. 2D vs. 3D) will be preferred by every patient. Our goal is to offer, within one tool, the widest possible range of approaches from which to choose from. This way the therapist will be able to test different options in the early phase of the treatment and decide which one represents the most suitable solution for the specific subject.

Elderly patients (or, in general, people with little familiarity with modern technology) may well refuse the 3D approach in the beginning, and switch to it later, after acquiring familiarity with the computerized therapy, while younger individuals, accustomed to playing videogames, will very likely prefer undergoing 3D exercises from the beginning.

\section{ACKNOWLEDGMENTS}

Our thanks to Prof. Giorgio Sandrini and Dr. Elena Sinforiani, from IRCCS C. Mondino Foundation, who collaborated on the development of the system and agreed to test it.

\section{REFERENCES}

[1] Abreu, P.F., Werneck, V.M.B., Costa, R.M.E. and Carvalho, L.A.V. 2011. Employing Multi-agents in 3-D Game for Cognitive Stimulation. Symposium on Virtual Reality (SVR), 2011 XIII, IEEE, (23-26 May 2011), 73-78.

[2] Anastasis. 2007. COG.I.T.O. http://cogito.integrazioni.it/ (last access: Feb 25, 2014)
[3] Dwolatzky T., Whitehead V., Doniger G. M., Simon E. S., Schweiger A., Jaffe D., Chertkow H. 2004. Validity of the Mindstreams computerized cognitive battery for mild cognitive impairment. Journal of Molecular Neuroscience. 2004, Vol. 24, Nr. 1, 33-44

[4] Eurostat. 2014. Computer use in individuals in EU countries. $\mathrm{http} / / /$ appsso.eurostat.ec.europa.eu/nui/show.do?dataset=isoc _ci_cfp_cu\&lang=en (last access: Apr 4, 2014)

[5] Eurostat. 2014. Individuals' level of computer skill in EU countries. http://epp.eurostat.ec.europa.eu/tgm/ refreshTableAction.do?tab=table\&plugin $=1 \&$ pcode $=$ tsdsc 46 $0 \&$ language $=$ en (last access: Apr 4, 2014)

[6] Fernández E., Bringas M.L., Salazar S., Rodríguez D., García M.E., Torres M. 2012. Clinical impact of RehaCom software for cognitive rehabilitation of patients with acquired brain injury. MEDICC Rev. 2012 Oct, 14(4):32-5.

[7] Giunti O. S. 2010. ERICA - Esercizi di RIabilitazione Cognitiva per Adulti. http://www.riabilitazione.giuntios.it/it (last access: Feb 25, 2014)

[8] Grau S., Tost D., Campeny R., Moya S., Ruiz M. 2010. Design of $3 D$ Virtual Neuropsychological Rehabilitation Activities. Second International Conference on Games and Virtual Worlds for Serious Applications (VS-GAMES) (2526 Mar 2010), 109 - 116

[9] Kueider A.M., Parisi J.M., Gross A.L., Rebok G.W.. 2012. Computerized cognitive training with older adults: a systematic review. PLoS One.

[10] Leonardi G., Panzarasa S., Quaglini S. 2011. Ontology-based automatic generation of computerized cognitive exercises. Stud Health Technol Inform. 2011, 169: 779-83.

[11] Rego P. 2010. Serious games for rehabilitation: A survey and a classification towards a taxonomy. 5th Iberian Conference on Information Systems and Technologies (CISTI) (16-19 June 2010), 1 - 6.

[12] Richardson M., Zorn T. E., Weaver K. 2002. Seniors' perspectives on the barriers, benefits and negative consequences of learning and using computers. http://www.academia.edu/download/31048190/resource1.pdf (last access: Apr 4, 2014)

[13] Rizzo A.S., Wiederhold B.K. 2006. Virtual Reality Technology for Psychological/Neuropsychological/Motor Assessment and Rehabilitation: Applications and Issues. IEEE Virtual Reality Conference, 2006 (25-29 Mar 2006), 308.

[14] Schuhfried. 1995. CogniPlus - I programmi di training. http://www.schuhfried.it/cogniplus-cps/i-programmi-ditraining/ (last access: Feb 25, 2014)

[15] Tornatore J.B., Hill, E., Laboff J.A., McGann M.E. 2005. Self-Administered Screening for Mild Cognitive Impairment: Initial Validation of a Computerized Test Battery. J Neuropsychiatry Clin Neurosci. 2005, 17(1), 98-105

[16] Tost D., Grau S., Ferre M., Garcia P., Tormos J.M., Garcia A., Roig T. 2009. PREVIRNEC: A cognitive telerehabilitation system based on Virtual Environments. Virtual Rehabilitation International Conference, 2009 (June 29 2009-July 2 2009), 87 - 93 